\title{
A practical approach to problems on return from deployment
}

\author{
MJ World
}

\section{Introduction}

There may be a latent period following return from an overseas deployment before certain diseases manifest themselves and soldiers should be made aware of this. Providing cards which, when presented, alert a future medical attendant to the possibility of a tropical disease (primarily malaria), is a simple precaution to take, particularly when the doctor concerned may have no experience of tropical diseases. The importance of continuing anti-malarial chemoprophylaxis for four weeks after leaving a tropical deployment should be emphasised to soldiers. Medical officers in temperate locations should not assume that the soldiers for whom they are responsible have all been in the United Kingdom for some time before deployment. It is not uncommon for soldiers in the Balkans to have deployed following relatively short periods of disembarkation leave after tours of duty in Belize, Brunei and, now, Sierra Leone where they may have acquired tropical diseases. Operational circumstances may prevent implementation of proper preventive measures. Observance of all the physical measures which can minimise the risk of insect bites may not be possible. Predeployment anti-malarial chemoprophylaxis may have been non-existent: it is just not practical to have battalions on such medications for long periods on the chance that tropical deployment may be necessary. Avoidance of immersion may be impossible with the consequence that risk of exposure to hantaviruses, schistosomiasis or leptospirosis is unavoidable.

It is impossible to anticipate every potential problem that may arise after tropical deployments. On the other hand, routine screening of asymptomatic individuals has not produced worthwhile returns for the effort involved. For example, no significant pathology was detected on screening the whole of the staff of a parachute field ambulance returning from a humanitarian mission in central Africa. Conversely, a single case of strongyloidiasis was identified when the urinary sediment was microscoped after persistent microscopic haematuria was detected. This case demonstrates the importance of following up findings discovered during routine medical examinations of military personnel who have served in the tropics.
In recent years, the author has been consulted on several occasions by symptomatic military personnel who have recently returned from tropical locations. In just about every case there has been a significant diagnosis. Not all have been primarily tropical diseases. Fortunately, none have been very serious. In some, the diagnosis has been made by a colleague but the patient has failed to respond to standard treatment. What are the options if confronted with falciparum malaria unresponsive to quinine?

To maximise the chances of successful diagnosis, a systematic approach is essential. Provided the nature and quality of biological samples are adequate, the reliability of the laboratory must be unimpeachable. Given these two requirements, it is unlikely that a significant diagnosis will be missed.

\section{Investigation protocol}

This is summarised in the Tropical Disease Investigation Sheet (see Table 1) which is the basis for systematic investigation of military patients where a tropical diagnosis is suspected.

\section{Personal details}

In the event that initial results suggest that further investigation is necessary, for example, corroboration of an unexpected result, it is essential to be able to contact the patient. Recording personal details will facilitate contact with personnel who may be posted frequently not only between units but also between deployments. Critical amongst these details is the travel history.

\section{Clinical diagnosis}

This appears at an early location and is the working diagnosis after taking a history and performing a physical examination. In essence, it is the shorthand summary of the case which determines the subsequent investigations.

\section{History of the present illness}

This should be succinct, enumerating the complaints and their duration with associated features. All drugs consumed should be recorded.

\section{Past tropical history}

The section prompts consideration of the main transmissible agents. Viruses, rickettsia, 
Table 1. Tropical Disease Investigation Sheet

\section{Personal Details:}

Surname:

Forename:

Rank:

Service:

Serv. No:

\section{Clinical Diagnosis:}

History of Present Illness:

\section{Past Tropical History:}

DoB:

Age (Yrs):

Hosp No:

Tropical Theatre(s) of Service:

\section{Date of Admission:}

Date of Discharge:
Viral:

Dengue:

Other:
Hepatitis:

Gastroenterology:

Malabsorption:

Peptic Ulceration:

Dysentery:

\section{Past General Medical History}

\section{Physical Examination}

General:

$\mathrm{Wt}(\mathrm{Kg})$ :

$\mathrm{Ht}(\mathrm{m})$ :

$\mathrm{BMI}(\mathrm{Kg} / \mathrm{m} 2)$

$\mathrm{T}(\operatorname{deg} \mathrm{C})$ :

Glands:
Alcohol:

Consumption:

Driving licence:

Withdrawal symptoms:

\section{Helminths:}

Strongyloidiasis

Schistosomiasis:

Other:
Giardia:

Amoebiasis:

Other:

\section{Smoking:}

Current:

Past:

Rash:

CVS: Pulse:

BP(lying):

$\mathrm{BP}$ (standing):

RS:

Abdomen:

CNS:

Urine $\mathrm{pH}$ :
Fundi:

Sensation to pinprick:

SG:
Reflexes:

Other:
Plantars:

Consultant psychiatric assessment:

\section{Investigations}

ESR:

$\mathrm{Hb}$ :

Indices:

WBC:

Diff: $\mathrm{N}_{-} \mathrm{L}_{-} \mathrm{M}_{-} \mathrm{B}_{-} \mathrm{E}$

Platelets:

INR:

MP Blood film:
Urea:

Creatinine:

$\mathrm{Na}$ :

$\mathrm{K}$ :

HCO3:

T.Prot:

Alb:
T.Bil:

AsT:

AIT:

yGT:

AIP

Glucose:

Ca:

Pi:
ECG:

Stools (x3)

CXR:

Abdo U/S:

Serology:

Amoebic FAT

Strong. ELISA

Other 
bacteria, protozoa and metazoa of medical significance are listed. Notably, fungi are not. Fungal diseases are rare other than in the immunocompromised but should not be entirely forgotten. Gastroenterological conditions, although not primarily tropical, are nevertheless more likely to be encountered as a consequence of foreign service and have both enteric and nonenteric sequelae. An example of a gastroenterological condition with enteric consequences after development of an infection-related complication is sterile congenital duodenal diverticula. These may remain entirely clinically silent until colonised by bacteria as a result of failure of gastric acid secretion after an episode of gastritis. The contaminated bowel syndrome which follows the hypochlorhydria may be particularly troublesome with malabsorption, diarrhoea and weight loss dominating the picture. The bacteria concerned (eg Escherichia coli) may well be commensals and considered non-pathogenic when confined to the colon but when established in the new location, disease results. An example of a gastro-enterological condition with a non-enteric complication is Reiter's syndrome. This condition with uveitis, arthritis, urethritis and sacroiliitis may be a late complication of dysentery due to Shigella or enteroinvasive Escherichia coli.

Alcohol consumption often increases during foreign postings. The duration of increased consumption may be insufficient to cause cirrhosis but continuation of high consumption on return may lead to road traffic accidents and consequent loss of driving licence. Conversely, withdrawal symptoms could occur if a precipitate reduction in rate of consumption after a prolonged period of high consumption was attempted. Fits, tremors and sweats could all be construed as arising from causes other than alcohol. An alcohol consumption history will alert the wary doctor to the possibility that symptoms are a consequence of excessive intake.

Smoking often increases during foreign postings. Cigarettes may be cheaper and smoking more accepted as a means of social intercourse than it is now in many western countries. Sometimes it is the only means whereby some rapport can be established with a suspicious and potentially hostile local militia with the result that symptoms arising from bronchial irritation by smoke arise acutely. In older soldiers, chronic excessive consumption may be sufficient explanation for right heart failure without having to invoke schistosomiasis or hydatid disease as possible causes even though exposure to these diseases may have occurred in the past.

\section{Past General Medical History}

Just because a military patient has been to an appropriate region does not mean the cause of their symptom is a tropical disease. Very often, western diseases are manifest for the first time on foreign postings. Whether this is due to environmental change, emotional stress or just coincidence is debatable but there may be evidence of antecedent disease even if a formal diagnosis has not been made. For example, it is worthwhile asking the patient with chronic diarrhoea arising apparently during a tropical deployment whether any self-limiting attacks occurred before being posted. It is not unknown for inflammatory bowel disease to commence in intermittent fashion before becoming persistent. Antecedent illnesses may predispose soldiers to tropical diseases. Too many who have had a splenectomy following abdominal trauma still manage to get posted to tropical areas.

\section{Physical examination}

The physical examination needs to be thorough if for no other reason than to exclude indicators for more extensive investigations than the routine investigation schedule. The general assessment is a good indicator of the severity of the case bearing in mind the stoical nature of soldiers in general and elite units in particular. It is the latter that are at greatest risk of tropical disease as the rapidity of deployment may preclude optimal preparation and the frequency of deployment increases risk. Weight on its own is of limited use. Height must be recorded as well in order to calculate body mass index (reference normal range $=20-25 \mathrm{~kg} / \mathrm{m}^{2}$ ), thereby providing a more objective measure of absolute nutritional status rather than just change in weight, important as the latter may be. Careful measurement of temperature using a reliable thermometer is essential. Although alternate day periodic fever (socalled "tertian" fever) is the classic chart in all forms of malaria apart from Plasmodium malariae where fever is separated by two afebrile days (so-called "quartan" fever), it is only a minority (about $8 \%$ ) of cases that exhibit this periodic pattern. Nevertheless, almost all $(96 \%)$ of cases of malaria have some kind of fever. Generalised lymphadenopathy occurs in a number of infectious diseases. Failure to detect this when present could have enormous implications if the case is human immunodeficiency virus infection and lymphadenopathy is the only sign. An indicator to perform an HIV test and provide timely treatment with beneficial effect on survival would have been lost. The full spectrum of rash distribution occurs in tropical diseases. The whole body, including genitalia, must be exposed and inspected carefully.

\section{Cardivascular Assessment}

Most antimalarial drugs are related to quinine which has effects on myocardial 
repolarisation manifest as a prolonged QT interval on the electrocardiogram. When corrected for cardiac rate (QTc) this should be less than $440 \mathrm{mSec}$. Prolongation is associated with arrhythmia. Certain combinations (eg halofantrine given after mefloquine in treatment dosage) can be particularly dangerous. Cardiac irregularity can also be caused by viral cardiomyopathies and pericarditis. Clinical evidence of cardiac failure may be apparent in the former and a pericardial rub may be heard in the latter. Postural hypotension will suggest hypovolaemia in the absence of any drug effect. Inordinate blood pressure fall on inspiration (pulsus paradoxus) will suggest pericardial effusion. All of these abnormalities indicate a need for echocardiography which is not part of the routine investigation schedule. Failure to look carefully for these signs will result in diagnostic delays and inappropriate or incomplete investigations.

\section{Respiratory System}

Clinical examination has been berated as most unreliable when compared with imaging methods. Certainly a chest X-Ray is included in the routine investigation for this very reason amongst others. Nevertheless, as a screening test for certain inflammatory conditions such as asthma and interstitial pneumonitis, it is still useful. Care should be exercised particularly when exercise tolerance is impaired. Post-exertional oxygen desaturation of haemoglobin may not be apparent clinically but a pulse oximeter may reliably indicate this giving an immediate indication for arterial blood gas analysis and high resolution CT scanning. Sputum microscopy must then be requested otherwise the presence of parasites such as lung flukes or nematode larvae may be missed.

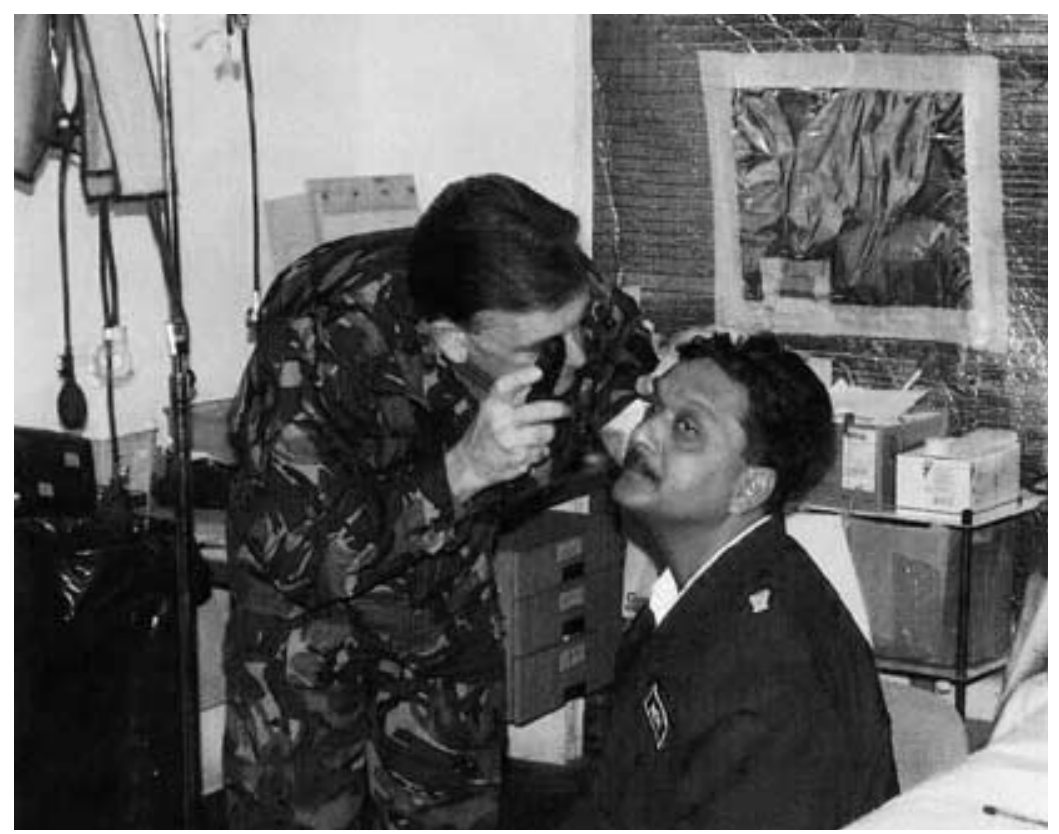

Fig 1. The author examining an Indian policeman with neurological symptoms who had returned to a temperate location after rest and recuperation in his home country.

\section{Abdominal Examination}

Lesser degrees of jaundice may be apparent only on abdominal inspection. This location is usually protected from the pigmenting effects of light. If jaundice is still in question it will heighten the need to pay attention to urinary findings and results of liver function tests. Audible bowel sounds (borborygmi) may announce bowel irritability and lower the threshold to request endoscopic assessments. Examination techniques for the spleen need to be good. Although abdominal ultrasound is a routine assessment modality, it is nevertheless useful to detect possible splenomegaly at an early stage in order to alert the ultrasonographer to assess splenic size and to consider the need for additional technology such as polymerase chain reaction-based detection of leishmanial DNA. Examination of the external genitalia is essential in order to diagnose undisclosed lesions related to sexual and other diseases. Although still in the minority, there will be an increasing requirement for expert gynaecological examination as the number of female military personnel grows. Routine vaginal swabs will be essential in females with fever.

\section{Neurological Examination}

This includes a psychiatric assessment in appropriate cases. Somatoform disorders have their origin in various stresses and even the most hardened military personnel are affected to some extent by the sights of remains of massacres and the results of mutilation particularly when they are not engaged in hostile activities. Any abnormality on a limited neurological examination of optic fundi (Figure 1), tendon reflexes, plantar responses and peripheral sensation to pinprick (remembering hypoaesthesia is more common than anaesthesia) should result in extension to a full neurological assessment.

\section{Urine}

The potential wealth of information provided by urinary examination dictates that dipstick assessment should never be omitted. Microscopic haematuria may be the only clue to the necessity to initiate multiple midday urinary examinations for schistosome eggs. Excess urobilinogen may be the only clinical indicator of haemolysis or minor hepatic disturbance.

\section{Investigations}

\section{Haematology}

Anaemia may be due to many causes but malaria and non-specific marrow depression are the main causes detected in soldiers. Low white cell counts are more commonly due to viral diseases but the effects of leishmaniasis and other causes of splenic enlargement should not be 
forgotten. Eosinophilia is a common finding during the migratory phases of helminthic diseases but in recent years eosinophilic gastroenteropathy, an idiopathic disease, and microscopic polyangitis, both uncommon non-tropical diseases, have been found to be the cause in caucasian soldiers. Eosinophilia in Gurkhas has been associated with presence of the dwarf tape worm (Hymenolepsis nana). A blood film for malarial parasites should never be omitted. Military laboratory technicians have more experience than most in assessing slides for this parasite.

\section{Renal Function Tests}

These are rarely found to be abnormal in soldiers returning from the tropics but do offer a reassurance that all is well provided serum creatinine is assessed in relation to age, sex and weight by one of the equations predicting glomerular filtration rate from these variables. In recent years, the Cockcroft-Gault equation (Male GFR= [140-age in years] $\mathrm{x}$ weight in kilogrammes/[creatinine in $\mathrm{uM} / 1 \mathrm{x} 0.814$ ) has achieved most widespread acceptance.

\section{Liver Function Tests}

Most cases seen in recent years of putative tropical disease have turned out to have abnormal liver function tests without disturbances of clotting with the result that a hepatitis is diagnosed.

\section{Calcium Metabolism}

This is rarely deranged but in occult sarcoidosis where there is the potential for hypervitaminosis $\mathrm{D}$, exposure to sunlight can cause a particular increase in calcium with consequent headache, polyuria and polydipsia.

\section{Electrocardiography}

Justification for this investigation has been given when discussing cardiovascular assessment (please see above).

\section{Stool Examination}

This is a basic examination and should never be omitted. Both microscopy and culture need to be undertaken and fresh stool specimens are essential in order to maximise the chance of identifying pathogens successfully.

\section{Chest Radiography}

A normal chest X-Ray is not only reassuring in a world where there is a global increase in the prevalence of tuberculosis but also provides a basis for comparison if respiratory symptoms should arise in the future. Fleeting pulmonary infiltrates may indicate the possibility of Loeffler's syndrome in those with eosinophilia or chronic organizing pneumonia of nontropical origin. Gurkhas have a lower threshold for contracting tuberculosis therefore this examination is particularly important in them.

\section{Abdominal Ultrasound}

This is particularly useful for assessing hepatic and splenic size and excluding amoebic and other abscesses in the liver. Cholelithiasis may occur in liver fluke infestations due to Clonorchis and Opisthoricis species but none have been diagnosed in soldiers in recent years. This position could change with the advent of sushi bars in the west and an acquisition of the taste for this form of food but the probability is fairly remote given the conservative British military attitude to food. Transrectal ultrasonography may be the technique of choice for assessing prostatic appearances in cases of possible prostatitis. The female generative organs are well demonstrated by transcutaneous and particularly by transvaginal ultrasound permitting better assessment of tubal and parametrial regions in cases of fever of unknown origin in female patients. In situations where doubt concerning ultrasonographic findings exists, computed $\mathrm{X}$-Ray tomography and magnetic resonance imaging techniques will be employed as ancillary assessment methods.

\section{Specific Serology}

There is a continuing extension of the investigational armamentarium as a result of greater routine availability of the techniques of molecular biology. The ability to amplify small amounts of DNA by the polymerase chain reaction with subsequent gene sequencing has enhanced diagnostic sensitivity whilst retaining specificity. Further refinements of this technology can be expected to enhance diagnostic capability. Sensitive enzyme-linked immunosorbent assays to detect specific antibodies still provide the main evidence of exposure to pathogens. Strongyloidiasis is notoriously difficult to diagnose and the life-threatening consequence of hyperinfection syndrome in the event that immunosuppresants have to be used make routine serological assessment for this pathogen mandatory. Similar considerations dictate the need for routine testing in relation to amoebiasis. Otherwise, serological assessments are dictated by other clinical and laboratory findings.

\section{Conclusion}

No serious tropical pathology should be missed if a standard investigational schedule is employed. Its sensitivity depends on the quality of the clinical and laboratory assessments and the experience of the staff with an ability to extend the investigational process when unexplained abnormalities are detected. On occasions, careful observation to assess subsequent 
developments may be preferable to further investigation and, ultimately, just as economical.

\section{Suggestions for further reading in relation to problems arising abroad}

Health Information for Overseas Travel, HMSO London 1995 (New edn in 2001)

Bell DR, Lecture Notes on Tropical Disease 4th Edn, Blackwell Science Ltd, Oxford 1995

Benenson AS, Control of Communicable Diseases in Man 16th Edn, American Public Health Association 1995

Cook GC (Ed), Manson's Tropical Diseases 20th Edn, Balliere Tindall, London 1996

Eddleston M, Pierini S, Oxford Handbook of Tropical

Medicine, Oxford University Press 1999
Mandal BK, Wilkins EGL, Dunbar EM, Mayon-White RT, Lecture Notes on Infectious Diseases 5th Edn, Blackwell Science Ltd, Oxford 1996

Salisbury DM, Begg NT (Eds), Immunisation against Infectious Disease, HMSO London 1996

Weatherall DJ, Ledingham JGG, Warrrell DA (Eds), Oxford Textbook of Medicine 3rd Edn, Vol 1 Section 7:

Infection, Oxford University Press 1996

\section{Addresses of some useful} websites

Centers for Communicable Disease Control and Prevention, Atlanta, Georgia, USA: http://www.cdc.gov/

Public Health Laboratory Service: http://www.phls.co.uk

World Health Organisation: http://www.who.int/ 